\title{
Análisis de las competencias docentes en proyectos de aprendizaje-servicio en la educación superior: La percepción del profesorado
}

Andresa Sartor-Harada ${ }^{1 *}$, Juliana Azevedo-Gomes ${ }^{1}$, Silvia Pueyo-Villa ${ }^{1}$ y Santiago Tejedor ${ }^{2}$

(1) Universidad Europea del Atlántico, Facultad Ciencias Sociales y Humanidades, Parque Científico y Tecnológico de Cantabria, Santander, España (correo-e: andresa.sartor@uneatlantico.es; juliana.azevedo@uneatlantico.es; silvia.pueyo@uneatlantico.es).

(2) Universidad Autónoma de Barcelona, Barcelona, España (correo-e: Santiago.Tejedor@uab.cat).

* Autor a quien debe ser dirigida la correspondencia.

Recibido Sep. 30, 2019; Aceptado Nov. 25, 2019; Versión final Ene. 8, 2020, Publicado Jun. 2020

\section{Resumen}

Este estudio analiza la percepción de los profesores respecto al desarrollo de competencias a partir de proyectos de el aprendizaje de servicio (ApS). ApS es una metodología activa en la que se combina aprendizaje académico y formación para la ciudadanía y la responsabilidad social. A pesar de que el enfoque de los proyectos suele estar en el aprendizaje de los alumnos, las experiencias en ApS permiten una construcción global y holística del conocimiento. Esta investigación, fundamentada en un paradigma mixto, ha aplicado un cuestionario semi-estructurado a 34 profesores de España, Portugal y diferentes países de América Latina que han participado en proyectos de ApS. Los resultados evidencian una percepción positiva de los docentes respecto a la adquisición de competencias relacionadas con el ApS. Sin embargo, el estudio advierte de la necesidad de formación específica para esta metodología en concreto.

Palabras clave: aprendizaje-servicio; proyectos; competencias; educación superior; docencia

\section{Analysis of teaching competences in service-learning projects in higher education: Teachers' perceptions}

\begin{abstract}
This study analyzes teachers' perceptions of competence development in service-learning (S-L) projects. S-L is an active methodology that combines academic learning and training for citizens and social responsibility. Although the focus of S-L projects is usually on the students' learning, experiences in S-L allow a global and holistic knowledge construction. This study was based on a mixed paradigm. A semi-structured questionnaire was applied to 34 teachers from Spain, Portugal and different Latin American countries that had previously participated in S-L projects. The results showed positive teacher's perception of skill acquisition related to SL. However, this study warns of the need for specific training for this particular methodology.
\end{abstract}




\section{INTRODUCCIÓN}

El aprendizaje de servicio (ApS) es una propuesta pedagógica innovadora que valora el aprendizaje en redes colaborativas. Tal y como apuntan Puig, et al (2007), existe un acervo variado de definiciones debido a que sus rasgos definitorios están presentes en diversas metodologías, como la educación ciudadana, el aprendizaje por proyectos, la integración de saberes o los servicios a la comunidad, entre otros. Los proyectos de ApS representan una acción solidaria protagonizada por los estudiantes, que tienen como objetivo atender, de manera eficaz, a las necesidades de la comunidad a través de una propuesta de trabajo fundamentada en los contenidos de aprendizaje previamente definidos (Briede y Mora, 2016). Son proyectos que, de manera simultánea, responden a la comunidad y a la calidad educativa. De este modo, el ApS permite un enfoque profesional y social que dota de nuevos matices y significados a los conocimientos de índole académica que adquieren nuevos valores como el respeto, el compromiso y, especialmente, la solidaridad (Tapia, 2006).

En la educación superior, el respaldo y la participación de las instituciones resultan decisivos para el éxito de las propuestas y proyectos de ApS. La apuesta por esta metodología demanda un trabajo formativo que confiera a las plantillas docentes un conjunto de competencias, habilidades y herramientas para su correcta aplicación. Pese a que el enfoque de los proyectos suele estar en el aprendizaje de los alumnos, las experiencias en ApS demuestran la capacidad de construir un conocimiento global construcción que impacta en las competencias docentes de forma tan intensa como en los educandos (Rodríguez, 2014). En este sentido, el hito de que la extensión universitaria, tanto entre el alumnado como en el profesorado, trabaje en el desarrollo de las competencias cívicas y cívico-sociales aseguraría una formación en valores para la vida en el conjunto de actores de la institución educativa (Alvariñas y Fernández-Villarino, 2011; Priegue y Losada, 2016).

Los antecedentes, teóricos e investigativos, sobre este campo han centrado sus esfuerzos en conocer las bases del ApS en la educación superior, así como la función docente y las competencias necesarias para actuar en este tipo de proyectos. No obstante, más allá de la necesaria definición de las competencias docentes que demanda esta metodología, apenas existen trabajos académicos que hayan analizado y comparado la percepción del profesorado respecto a las competencias desarrolladas durante los proyectos de ApS. En este sentido, este estudio ha perseguido dos objetivos. Por un lado, el análisis y la descripción de cómo los docentes perciben su propia adquisición de competencias en el marco de proyectos de ApS en la educación superior; y por otro, la detección de las principales dificultades encontradas en este proceso.

La metodología ApS emerge en un momento socioeducativo caracterizado por la voluntad de conferir un mayor protagonismo al alumnado dentro de proyectos que permitan asociar conocimientos de varias áreas. La transformación del escenario informativo y comunicativo (Rodrigues y Zárate, 2018) por el impacto de la tecnología ha alcanzado igualmente a los procesos educativos. Los contenidos se han transformado en piezas portátiles, personalizadas y participativas, y el alumnado demanda un protagonismo mayor en su formación. En esta línea, Furco (2002) asevera que la mixtura entre comunidad y academia son los ingredientes decisivos.

El aprendizaje servicio pretende comprometer a los individuos en actividades que combinan servicio a la comunidad y aprendizaje académico. Dado que los programas de aprendizaje servicio normalmente están radicados en cursos formales, la actividad de servicio habitualmente se basa en los contenidos del currículum que se enseñan. Este vínculo entre lo académico y el desarrollo de la ciudadanía está adscrito al paradigma de la complejidad (Morin, 2007), que considera que la educación transciende los contenidos curriculares y debe integrar saberes de varias áreas. Así, el aprendizaje de servicio nace como una opción metodológica activa, que provoca la interrelación de diferentes saberes y fomenta la construcción de un conocimiento colectivo con la generación de un producto final beneficioso para la comunidad.

\section{OTROS ANTECEDENTES}

En el contexto latinoamericano, especialmente en países como Argentina, Chile, Uruguay y Brasil, las prácticas de ApS acumulan un extenso recorrido desde los años 80 e incorporan además la solidaridad como un valor añadido al servicio aplicado a las comunidades participantes (Cecchi, 2006). De ahí que se le denomine pedagogía del "aprendizaje de servicio solidario" más que aprendizaje servicio. La creación en 2002 del Centro Latinoamericano de Aprendizaje y Servicio Solidario (CLAYSS), con sede en la capital federal de Buenos Aires, ha constituido un hito decisivo en este proceso de transformación educativa. En el contexto europeo, las primeras iniciativas formales de ApS se vinculan al desarrollo de diversos foros y conferencias, como el Fórum Cívico-Educativo de Madrid en 2005, las iniciativas de la Red Universitaria Española de Aprendizaje-Servicio (ApS-U), el IX Congreso Español y el I Europeo de Aprendizaje-Servicio en Educación Superior, celebrados en Madrid, en el año 2018 y las conferencias internacionales en ApS para la formación docente, celebradas en Bélgica, en 2007; y en Irlanda, en 2008 (Folgueiras, Luna González y Puig, 2013). 
Las propuestas académicas de ApS en la educación superior responden al nuevo modelo formativo de la universidad, que impulsa la necesidad de combinar el aprendizaje académico con la formación para la ciudadanía y la responsabilidad social (Rodríguez, 2004; Vallaeys, 2014; Larrán-Jorge y Peña, 2015; Dolgon y Eatman, 2017). Actualmente, las universidades intentan responder y, a la vez, adaptarse a los nuevos tiempos de la tan valorada innovación curricular, la educación para la ciudadanía y el desarrollo de las competencias sociales (Gil-Gómez, Molines-García, Chiva-Bartoll y García-López, 2016; Martínez Dominguez, Martínes Dominguez, Alonso y Gezuraga, 2013; Martínez, 2010; Tryon, Sotecker, martin, Seblonka, Hilgerndorf y Nellis, 2008). Para Santos Rego y Moledo (2018) y Wall y Stanton (2018), estos proyectos son una oportunidad de formación en una dimensión holística, pues permiten abarcar sensibilidades y emociones, llegando incluso a la misma conciencia de los futuros egresados como seres eminentemente sociales.

Las instituciones de educación superior atienden a poblaciones de estudiantes muy diversas y multiculturales. El desarrollo de la tecnología ha permitido progresivamente que las personas accedan a la universidad en diferentes momentos de su vida. Este nuevo perfil de alumno demanda nuevas metodologías de enseñanza, que fomenten el desarrollo de conocimientos y que les permitan implementar habilidades y competencias curriculares y profesionales (González-Aldea y Marta-Lazo, 2015; Marta-Lazo y González-Aldea, 2012; Cabanach, Moll y Rodrigo 2009; Basinger y Bartholome, 2006). Un porcentaje elevado de la población no sigue sus estudios al finalizar la secundaria, sino que los hace después de un período laboral, teniendo que armonizar estudio y trabajo o respondiendo a formaciones continuas a lo largo de toda la vida. En este sentido, los centros de educación superior se preparan para acoger este nuevo perfil de estudiantes, con sus diferentes objetivos y expectativas (De Miguel, 2005; Shurman y Ballon, 2016). En este contexto, los involucrados en proyectos de ApS conciben el servicio como una respuesta a las necesidades reales de la sociedad a la que pertenecen y destacan la preservación y la recuperación del medio ambiente como una medida necesaria en este espacio de colaboración recíproca (Gelmon y Spring, 2018). Existe una amplia amalgama de posibilidades, como la recuperación del patrimonio cultural, la ayuda en centros educativos, la colaboración con grupos sociales que presenten necesidades especiales o el fomento de campañas de sensibilización, entre otros (Puig et al, 2007).

El ApS implica el desarrollo simultáneo de enfoques pedagógicos y solidarios en la formación de las personas involucradas (Zabalza, 2004; Tapia, 2006; Zaitseva y Ersozlu, 2017). De este modo, la educación se convierte en un proceso de asimilación de compromisos con intencionalidades altruistas y en función del bien común. El compromiso con el servicio público y la responsabilidad social que sugieren la metodología ApS garantizan la formación holística del alumnado involucrado (Carbonell y Carrillo, 2008). Por otra parte, el profesorado promueve el aprendizaje cívico de manera significativa (Howard, 2001). Las propuestas deben combinar procesos o dinámicas necesarias para la comunidad que, a partir de proyectos correctamente articulados, fomentarán el desarrollo de la responsabilidad social. Así, los participantes trabajarán en función de las necesidades reales del contexto y con el determinante objetivo de mejorarlo (Puig et al., 2007).

Los saberes y las aptitudes que desarrollan los proyectos ApS responden, además, a las nuevas competencias determinadas por el "Proyecto Tuning Latinoamericano", que incorpora la responsabilidad social, el compromiso ciudadano, la salvaguarda del entorno socio-cultural y la preservación del medio ambiente como las principales bases para mejorar la colaboración entre instituciones de educación superior y para el fomento del desarrollo de la calidad, la efectividad y la transparencia en la educación (González, Wagenaar y Beneitone, 2004). A partir de este compromiso ciudadano, Priegue y Losada (2016), en sus trabajos e investigaciones, Ramírez y Pizarro (2005) y Daynes y Longo (2004), en sus materiales para la formación docente, identifican como fundamental la adquisición de competencias cívico-sociales y el desarrollo de la sensibilidad frente a las necesidades de la comunidad en la que se establece y se desenvuelve el proyecto. En este contexto, el compromiso cívico favorece el impacto y la repercusión social a nivel comunitario (Kaye, 2004; Puig et al., 2007). Cano (2012) y Arifin (2015) definen la competencia como el conjunto de conocimientos, actitudes y habilidades que se relacionan y permiten el desarrollo profesional. Por su parte, Kumpulainen y Lankinen (p. 75, 2016), añaden que, más allá de la ejecución de tareas, "la competencia es una característica del operador que le permite dar una significación a la situación de trabajo".

Por lo que respecta al rol docente en los proyectos de ApS, Villa y Poblete (2008) apuntan que el papel activo del docente permite el desarrollo de competencias y además la integración de aprendizajes ya existentes, con el propósito de "invertir el bagaje universitario, personal y técnico en la construcción de estructuras sociales más humanas" (Villa y Poblete, 2008). Con relación a ello, de acuerdo con Cano (2012) y Navarro y Sampson (2016), el buen profesor es el que es capaz de reflexionar sobre la acción e integrar todos los conocimientos, actitudes y habilidades para tomar decisiones o dar respuesta a las situaciones pedagógicas que se presentan. Partiendo de ello, el análisis del profesorado sobre su propia acción docente y la detección de las competencias adquiridas en su acción pedagógica conforman una reflexión que permite establecer puentes 
entre conocimientos existentes y nuevos (Canquiz, 2010; Bergsmann y Spiel, 2015). En el caso del ApS resulta fundamental que el profesor sea consciente de las competencias que adquiere y de aquellas que puede promover en el alumnado mediante la realización de estos proyectos. Por esta razón, la percepción que tienen los profesores universitarios sobre las competencias adquiridas durante el proyecto de ApS constituye un aspecto decisivo en el conjunto del proceso educativo.

\section{METODOLOGÍA}

La presente investigación sobre la metodología de ApS en la educación superior pone de manifiesto la necesidad de conocer las creencias de los profesores universitarios respecto al desarrollo de competencias, así como la importancia de identificar las dificultades encontradas durante su participación en este tipo de proyectos. Para ello, se optó por un diseño de investigación descriptiva con un enfoque mixto y de tipo ex post facto.

En consonancia con las vertientes cualitativa y cuantitativa que conducen este estudio (Hernández-Sampieri, 2010), el instrumento elegido para la recogida de información ha sido un cuestionario semiestructurado, construido con preguntas abiertas y cerradas, que busca la elaboración de una diagnosis, a modo de perfil, de los docentes participantes. Además, esta herramienta ha permitido la identificación de sus creencias sobre las competencias adquiridas en el proyecto de ApS y las dificultades encontradas en este proceso. Partiendo de ello, el cuestionario fue diseñado a partir de tres dimensiones (ver Fig. 1):

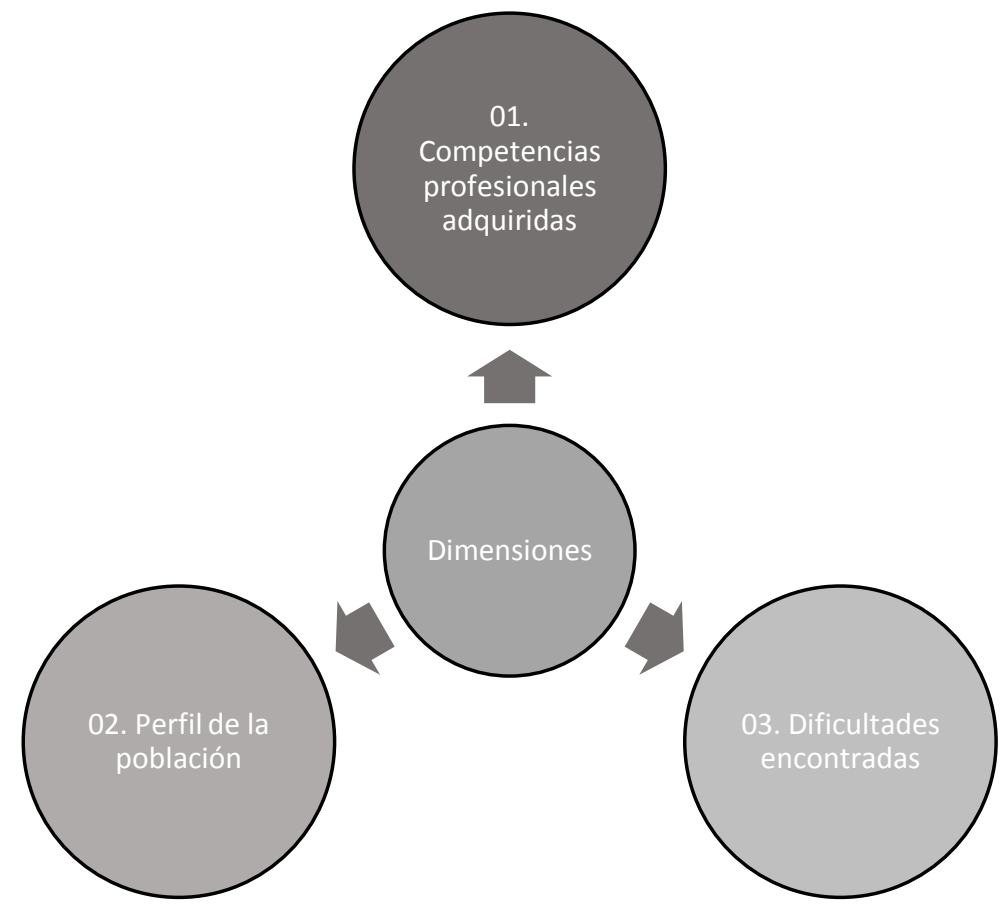

Fig. 1: Dimensiones del cuestionario semiestructurado.

Para la validación de contenido del cuestionario, se seleccionó a un grupo de expertos integrado por dos docentes del área de formación del profesorado y un profesor del área de metodología de la investigación científica de la Universidad Europea del Atlántico. A los tres expertos se les entregó un documento con los objetivos del cuestionario y su contenido. Asimismo, se les informó sobre cómo debían valorar las preguntas planteadas en conformidad con el objetivo del instrumento. Después de la revisión, el $100 \%$ de los expertos coincidió en mantener las preguntas y sus respectivas escalas de valoración, tras realizar solamente pequeños ajustes de estilo lingüístico en algunas preguntas. Por esta razón, no se consideró necesario calcular un índice de validez de contenido.

En este sentido, con el propósito de conformar una muestra intercultural, con amplia variedad de perfiles que permitieran contrastar distintos puntos de vista en relación con su entorno profesional, la población del estudio se conformó a partir de las respuestas a un anuncio en la red social Linkedin, en el que se buscaban docentes: a) que actuaran en la educación superior o b) que hubieran participado en algún proyecto de ApS en el entorno universitario. El anuncio fue publicado en diciembre de 2018, en castellano y en portugués, con el objetivo de alcanzar profesionales de todos los países de América Latina, España y Portugal. El cuestionario se elaboró en formato digital y se aplicó a través de la plataforma de Google durante los meses de enero a marzo de 2019. 
En un primer momento, se recibieron 53 respuestas al cuestionario. Tras un primer análisis de la respuesta a la pregunta "Explique brevemente el tipo de proyecto en Aprendizaje Servicio en que participaste", se detectó que el $36 \%$ de los profesores encuestados no había participado en un proyecto propiamente de ApS, sino de de otra naturaleza. Por esta razón, con el propósito de analizar de manera fehaciente las percepciones del profesorado sobre las competencias adquiridas en proyectos de ApS, se descartaron sus respuestas.

Finalmente, la muestra estuvo conformada por 34 docentes: 23 docentes universitarios provenientes de países hispanohablantes y 11 docentes universitarios, de países luso hablantes. Concretamente, la investigación contó con la participación de docentes de Brasil, Ecuador, Honduras, Argentina, Perú, Paraguay, Guatemala, Uruguay, Colombia, México, España y Portugal. Los proyectos se han centrado en las siguientes temáticas:

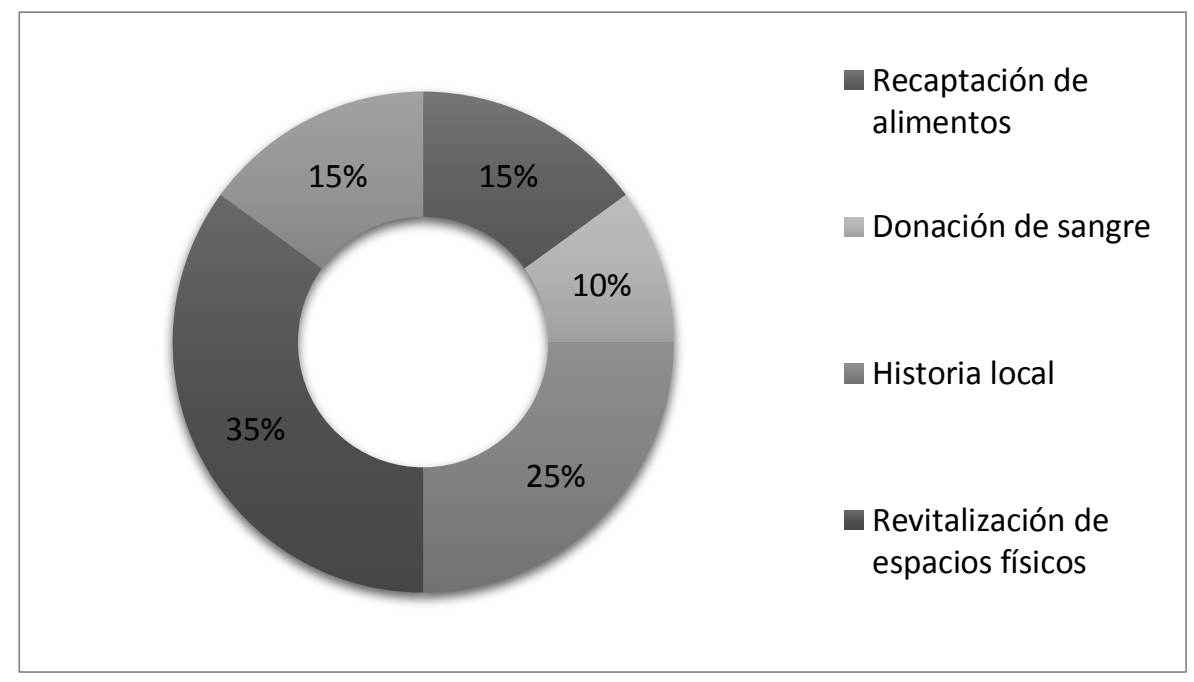

Fig. 2: Áreas temáticas de los proyectos de ApS

Para el tratamiento y análisis de la información se optó por la categorización temática, seguida de la técnica de análisis de contenido (Bardin, 1991), a fin de identificar en el discurso de los docentes las competencias que consideran haber adquirido y las dificultades encontradas en su participación. Para esta segunda etapa de análisis se utilizó como soporte el software Atlas-Ti (Versión 8).

La investigación se ha fundamentado en una metodología de trabajo híbrida basada en una encuesta matriz que cruzaba preguntas de tipo cuantitativo con cuestiones abiertas que han permitido un trabajo más cualitativo por parte de los docentes en la lectura e interpretación de las respuestas de los alumnos. A través de este tipo de cuestionarios cerrados se ha analizado, entre otras, las siguientes cuestiones: a) formación inicial; b) conocimiento acerca de la participación y roles en proyectos de ApS; c) competencias desarrolladas en proyectos ApS, d) responsabilidad social, compromiso ciudadano y preservación del medio ambiente contemplados en los proyectos ApS. Se han aplicado diferentes cuestionarios, así como el cruce de los resultados obtenidos en ellos y la creación de nuevos listados de preguntas. Los datos cuantitativos se han analizado a partir de frecuencias y de porcentajes. Las variables principales de las preguntas de los cuestionarios han sido las competencias técnicas, socio-emocionales y organizativas contempladas por el proyecto en el que han participado los sujetos.

Los cuestionarios, de 20 preguntas cada uno, se han utilizado como herramienta metodológica principal, la cual ha posibilitado la definición de diferentes indicadores a partir de las respuestas obtenidas. En este sentido, se han incorporado preguntas abiertas y cerradas. La propuesta metodológica incluye preguntas cerradas dicotómicas para los temas más definidos sobre el grado de desarrollo de las competencias previstas en los proyectos de Aprendizaje y Servicio.

\section{RESULTADOS Y DISCUSIÓN}

El análisis de los cuestionarios realizados a expertos permite extraer una serie de resultados en diferentes ámbitos. Por un lado, respecto a la percepción del profesorado participante sobre las competencias adquiridas, se detectaron tres grandes categorías de competencias: competencias socioemocionales (CS), competencias organizativas (CO) y competencias técnicas relacionadas con el tema del proyecto (CT). Además, cada categoría se dividió en tres o dos subcategorías. A continuación, se procede al análisis y sistematización de las reflexiones derivadas de cada una de las categorías competenciales (ver Tabla 2). 
Tabla 2: Categorías competenciales.

\begin{tabular}{|l|l|l|l|l|l|l|}
\hline Categoría & \multicolumn{2}{|l|}{ CS } & \multicolumn{2}{l|}{ CO } & \multicolumn{2}{l|}{ CT } \\
\hline Meta categoría & $\begin{array}{l}\text { Competencias } \\
\text { emocionales }\end{array}$ & $35 \%$ & $\begin{array}{l}\text { Acompañamiento y } \\
\text { seguimiento de } \\
\text { proyectos en } \\
\text { general. }\end{array}$ & $41 \%$ & $\begin{array}{l}\text { Desarrollo del } \\
\text { conocimiento } \\
\text { teórico-práctico }\end{array}$ & $15 \%$ \\
\cline { 2 - 6 } & $\begin{array}{l}\text { Sensibilización } \\
\text { con la } \\
\text { comunidad }\end{array}$ & $12 \%$ & $\begin{array}{l}\text { Trabajo en equipo } \\
\text { y Liderazgo }\end{array}$ & $21 \%$ & $\begin{array}{l}\text { Conocimientos } \\
\text { técnicos } \\
\text { específicos del } \\
\text { proyecto }\end{array}$ & $26 \%$ \\
\cline { 2 - 7 } & Empatía & $21 \%$ & & & & \\
\hline
\end{tabular}

\section{Competencias socio-emocionales (CS)}

Los resultados derivados del análisis de las competencias socioemocionales, concebidas como aquellas que favorecen las interacciones y la resolución de problemas por medio de la actuación ética en diversos contextos (Goodman y Tyler, 2015), inciden en la importancia de la implicación del profesorado en el contexto, así como en la necesidad de interrelacionarse con varios agentes en el proyecto de ApS. De este modo, un $68 \%$ del profesorado participante cree haber adquirido este tipo de competencias. En esta categoría, se destacan como principales competencias:

Competencias emocionales: El trabajo en proyectos de ApS exige que el docente se implique con el contexto y con los participantes. Por esta razón, los docentes manifestaron el desafío y el aprendizaje obtenido en trabajar con personas en un contexto más allá del aula. El profesor comparte su conocimiento y asume un papel de coordinador, rol al que no siempre está acostumbrado.

P12: "La construcción del proyecto nos pone a prueba en todo sentido, desde lo emocional, teórico y en lo vincular. Se aprende sobre todo a superar la incertidumbre y a construir conjuntamente hacia un resultado compartido". Especialmente en la educación superior, el profesorado debe ser capaz de atender los diferentes intereses y necesidades de los estudiantes (Priegue y Losada 2016). Una vez que los alumnos demuestren opiniones y modos propios de actuar, exigirán sensibilidad y flexibilidad por parte del profesor para negociar las distintas acciones que demanda un proyecto de ApS. Además, los profesores ratifican que, por ser un proyecto de carácter práctico, se pone de manifiesto una responsabilidad y un encuentro con la realidad que provoca un cambio importante de percepción.

P3: "Prevenir que nuestros niños sean presa fácil para inducirlos en la delincuencia, reclutamiento para el ingreso a las pandillas, embarazo en adolescentes, etc. pues ese es el ambiente que se vive en estas comunidades como la nuestra, violencia me ha provocado un cambio de visión y de actitud hacia los adolescentes de mi centro". Sensibilización con la comunidad: La participación como docente en un proyecto que debe provocar un cambio en la comunidad exige que se perciba y se reflexione sobre las necesidades de este entorno. Los profesores participantes apuntan que el proceso ha modificado su manera de percibir esta realidad.

P.5: "Es que crees que conoces al centro y a la comunidad. Pero no es así. Cuando empezamos, yo no era consciente de las dificultades con las que convivían mis alumnos. Al empezar a trabajar con el tema de la higiene bucal, saltaron tantos problemas más que es imposible que no te impliques. Este fue mi primer proyecto de aprendizaje-servicio y sin duda, el más impactante. Me tocó tanto, que ahora mi principal tarea es buscar apoyo de instituciones que puedan participar en los proyectos y aportar, ni que sea un poquito, a esta comunidad".

P.17: "Creo fue muy positivo, sobre todo porque los profesores que participamos adquirimos mayor conocimiento y sensibilidad frente a la situación económica y social que viven las comunidades rurales, pero también, porque fuimos útiles a la comunidad y ganamos habilidades para interacción directa con personas humildes de nuestra región". Los profesores universitarios no pocas veces trabajan en más de una institución y no necesariamente están implicados en la comunidad donde se realizará el proyecto de ApS. La consciencia demostrada por los profesores sobre la importancia de conocer las necesidades de la comunidad en que se realizará el proyecto (Puig et al., 2007) es un indicador para la formación docente en proyectos de ApS. Los profesores participantes también coinciden en señalar el importante grado de satisfacción personal derivado de su participación en el proyecto. Este aspecto está directamente vehiculado con los beneficios aportados a la comunidad y al centro. 
P.26: "Uno de los beneficios que vi reflejado en la comunidad fue el producto final del proyecto, con la plantación de árboles en conjunto con las familias. A partir de este proyecto, los demás profesores también se interesaron y yo me siento feliz en haber participado de este primer paso". Empatía: Vinculada a la sensibilización con la comunidad, el término empatía, del griego $\varepsilon \mu \pi a ́ \theta \varepsilon ı$ (cualidad de sentirse dentro) ha tenido diferentes significados, tanto desde el enfoque cognitivo como desde el afectivo. Sea para imaginarse en el lugar del otro, sea para comprender la representación que hacen los demás (Schaffer, 2002), en los proyectos de ApS, los profesores se reconocen involucrados con la propuesta y afirman que interactúan con la comunidad. Por esta razón, la empatía aparece identificada como una de las características desarrolladas en el marco de su experiencia en proyectos de ApS.

P.16: "Desde antes de participar en esta experiencia ya tenía una sensibilidad y compromiso social, pero al conocer de primera mano la situación y forma de vida de las comunidades rurales pobres, se reafirmó mi intención de tratar de aportar a la solución de sus problemas; de allí en adelante siempre he tratado de hacer los diferentes trabajos por los que he pasado teniendo en cuenta el contexto social y económico que vive mi país y tratando de sensibilizar a las personas con las que he interactuando para que en conjunto y desde lo que realiza cada quién, aportemos a la transformación del mundo". Otros participantes detallan los cambios de actitudes derivados del desarrollo de la empatía que consideran haber adquirido en su trabajo con el proyecto de ApS.

P.30: "Desde que comencé mi formación en docencia empezó un proceso de transformación personal y desde entonces, me he empeñado en comprender cada vez más, cómo funcionan los grupos sociales. Por esto, siempre procuro promover debates sobre temas al respecto; sobre los gobiernos, las creencias, la cultura, la actitud de las personas. El planteamiento, con la intención de conocer las perspectivas de las personas y en el proceso, con la intención de un aprendizaje mutuo es un gran aprendizaje". La empatía es una competencia emocional que algunos docentes han adquirido y/o potenciado durante la implementación del proyecto y, además, han podido trabajar también con sus estudiantes. Estos pronto se insertarán al mercado laboral y el desarrollo de esta competencia les ayudará a realizar una mejor gestión emocional de las relaciones profesionales en el ámbito laboral.

\section{Competencias Organizativas (CO)}

Un $62 \%$ del profesorado considera haber adquirido competencias organizativas en su experiencia dentro de proyectos de ApS. Las competencias organizativas se centran en administrar los recursos de la escuela, coordinar y organizar las posibilidades del componente humano de la comunidad educativa. De este modo, planificar, organizar, gestionar y liderar, son acciones necesarias en los proyectos de ApS. El profesorado ratifica estas acciones y reconoce haber realizado tareas organizativas en diferentes momentos del proyecto. Acompañamiento y seguimiento de proyectos en general: el profesorado afirma haber desarrollado diferentes competencias relacionadas con la organización y la gestión.

P4: "Ha mejorado mis competencias de comunicación, organización, evaluación, trabajo en equipo o gestión".

P9: "La gestión, la coordinación, la orientación de grupos, la investigación y el abordaje del marco teórico necesario para sostener la práctica, la rigurosidad en la organización, todo esto lo desarrollé". Trabajo en equipo y Liderazgo: el liderazgo, como conjunto de habilidades directivas para influir en un grupo de trabajo, no es un tema menor en el rol docente en un proyecto de ApS. Los participantes identifican como un desafío la tarea de gestionar personas en la medida en que constituye una propuesta distinta a un proyecto en el aula.

P20: "Aprender a trabajar con las comunas, que son agrupaciones de barrios en mi país, entendiendo que ellos participan, a través de sus líderes, de las decisiones como en qué se invierte el dinero que les asigna la alcaldía municipal para sus proyectos". La principal diferencia entre un proyecto temático común y un proyecto de ApS es la implicación de diferentes agentes, como familiares y miembros de la comunidad, que asumen distintos roles, pero que, a la vez, inciden directamente en el proyecto. Este conjunto de elementos debe ser gestionado por el docente, aspecto que puede ser concebido como como un reto y también como un nuevo aprendizaje.

P.2: "Es que me tuve que involucrar, y dirigir y pensar en todos los implicados. Fue mucho más allá de lo que estaba acostumbrado a realizar en mi aula. Ahora me siento mucho más capaz de trabajar con grupos".

P.28: "Lo que más aprendí fue a gestionar diferentes grupos con el mismo objetivo. Esto es un aprendizaje que llevaré para los demás proyectos, porque me costó mucho a principio y ahora me veo más capaz".

P.14: "El hecho de trabajar con personas ajenas al centro educativo me exigió mucho más, porque no estaban a mi cargo, sino ayudándonos a cambiar el proceso de recogida de basura alrededor del centro. Yo tuve que 
aprender a gestionar no solo la planificación del proyecto, sino las personas en todo momento". Las competencias organizativas son necesarias no solamente para proyectos de ApS, sino también para las labores docentes en diversos ámbitos de actuación. En la formación docente inicial no siempre se trabaja suficientemente esta competencia. Esto conlleva que los profesores universitarios sean capaces de gestionar bien sus acciones docentes, pero tengan dificultades para gestionar las acciones grupales por parte de sus estudiantes. Por esta razón, los profesores mencionan que el trabajo con proyectos de ApS les ha obligado a desarrollar estas competencias que traspasan la competencia didáctica que se trabaja en el aula. (Santos Rego et al, 2018).

\section{Competencias Técnicas (CT)}

Las competencias técnicas son aquellas que están relacionadas directamente con conocimientos y habilidades específicas para el desarrollo del proyecto de ApS. En los proyectos de ApS poseen una importancia destacada, ya que se integran aprendizajes ya existentes y se construyen nuevos aprendizajes (Villa y Poblete, 2008). Desarrollo del conocimiento teórico-práctico: algunos participantes resaltan haber aprendido sobre las temáticas específicas trabajadas en los proyectos.

P.16: "Conocimiento, organización y Orientación de la Pedagogía en ambientes no convencionales (Contextos hospitalarios - Pedagogía hospitalaria), (competencia organizacional)".

P.28: "Adquirí nuevos conocimientos, especialmente como compromiso social y nutricional porque no solo se le enseña al niño a valorar lo que se produce en la comunidad, sino que también la manera como se debe de promover la producción y consumo de productos naturales para mejorar nuestra salud, encima también se enseña la comercialización y a mejorar la economía familiar".

P.23: "Aspectos en la organización de la actividad, el papel de orientador en el montaje del proyecto, apropiación de temas nutricionales por medio de investigación". Conocimientos técnicos específicos del proyecto: otros profesores destacan algunas competencias técnicas y pedagógicas adquiridas que, aunque están menos relacionadas con el proyecto en sí, son aprendizajes que facilitarán su labor docente en el futuro.

P.7: "El uso de equipos tecnológicos en trabajo de comunas, es también algo nuevo para mí, ya que lo requerían las asignaturas a mi cargo".

P.9. "He adquirido competencia en tutoría, en acompañamiento y seguimiento. En aprender a redactar los informes de los estudiantes de forma concisa y exacta, respetando al estudiante tal cual es, evitando reflejarme y permitirles sean ellos mismos". El aprendizaje de otros contenidos curriculares y de otras destrezas docentes se relaciona con el paradigma de la complejidad (Morin, 2007). Este paradigma considera la combinación de saberes, competencias y habilidades como elementos que se retroalimentan en la sociedad del conocimiento. A partir de esta adquisición de competencias sentida pelos profesores, se puede afirmar que los proyectos de ApS, por tanto, responden a la vinculación entre teoría y práctica, entre lo académico y lo comunitario y complementa los saberes didácticos que se aplican en la universidad.

\section{DIFICULTADES ENCONTRADAS}

Las principales dificultades señaladas por los docentes (ver Fig. 3) aluden, por un lado, a la falta de formación específica en relación con el desarrollo de proyectos de ApS; y por otro, a la falta de respaldo por parte de su institución. Asimismo, el profesorado alude a una tercera problemática, pero en menor medida. Se trata de la posibilidad de acceso e interacción con las comunidades que participaban de las iniciativas.

La falta de formación específica ha sido identificada como un problema por más del $63 \%$ de los participantes. A pesar de que consideran muy positivas sus participaciones y reconocen la asimilación de diferentes competencias, los docentes creen que la falta de formación es la principal debilidad o flaqueza en el desarrollo de los proyectos de ApS. Al analizar las respuestas relacionadas con la adquisición de competencias, es posible constatar que la formación necesaria para la realización de proyectos de ApS como docentes universitarios traspasa la formación metodológica, es decir, de cómo aplicar los proyectos, sino que debe incluir todos los elementos no académicos que se deben considerar, como, por ejemplo, las competencias socioemocionales, organizativas y técnicas, necesarias para el mejor desarrollo de proyecto.

P.2: "Si hubiéramos recibido algo de capacitación previa sobre cómo organizar todas las fases del proyecto, habríamos finalizado antes y con resultados mejores. Yo me sentí responsable por los estancamientos vividos con mis alumnos de nutrición". 


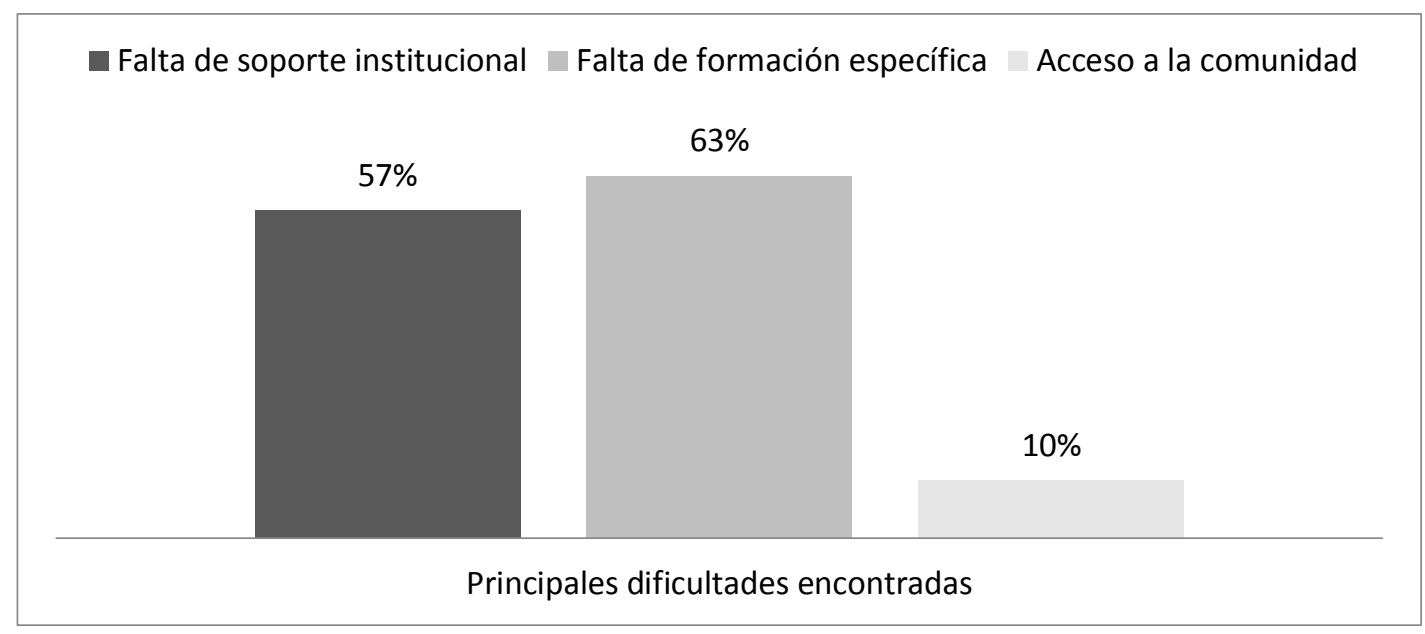

Fig. 3: Resumen de las dificultades encontradas por los docentes en los proyectos de ApS.

P.21: "La falta de formación, ya que no existe una escuela que nos forme como gestores ante las comunidades, nuestro desarrollo se lleva a cabo por interés personal y la integración de estudiantes a un área llamada atención a comunidades, donde pueden trabajar como parte de su servicio social. La universidad cree que estamos preparados para lidiar con estos aspectos más organizativos, pero no es así, a mí me costó muchísimo". Si el papel de la universidad es favorecer la integración de aprendizajes académicos con la formación para la ciudadanía (Jorge y Peña, 2015), las instituciones adquieren una responsabilidad importante a la hora de fomentar y apoyar los proyectos de ApS realizados. No obstante, la falta de apoyo por parte de la institución no es un tema menor en las dificultades encontradas por los profesores. Este tema ha sido mencionado por el $57 \%$ de los participantes en el estudio. En general, la falta de apoyo es financiero y logístico, lo que limita el alcance de la propuesta.

P.1: "Falta de recursos para transporte de estudiantes, a veces falta de colaboración de productores. Falta de tutores técnicos docentes".

P.33: "La dificultad grande fue la falta de apoyo económico y logístico por parte de la institución universitaria a la que pertenecíamos. La logística y el presupuesto del proyecto fue muy limitada pues todo se cubría con recursos propios de los estudiantes y de las comunidades campesinas locales. Hizo falta una mayor preparación en cuanto a materiales didácticos que se hubieran podido diseñar y llevarle a los campesinos". Asimismo, el hecho de trabajar con comunidades también ofrece retos, como menciona el $10 \%$ de los participantes.

P5: "Los contextos de intervención en algunos casos cuentan con restricciones de acceso". Las evidencias recogidas ponen de manifiesto el vínculo entre las competencias que los profesores consideran haber adquirido durante su participación en los proyectos de ApS. Las competencias socioemocionales y el desarrollo de la sensibilidad frente a las necesidades de la comunidad (Priegue y Losada, 2016) es un elemento de gran valor. Por otra parte, los docentes destacan la importancia que posee la adquisición de competencias relacionadas con la organización, como por ejemplo el trabajo en equipo y la organización del proyecto en general.

\section{DISCUSIÓN FINAL}

La presente investigación tiene como objetivo identificar la percepción de los docentes de educación superior sobre las competencias adquiridas y las dificultades encontradas en su participación en proyectos de ApS. A partir del estudio, es posible concluir que:

1) El profesorado incide en el deficiente apoyo institucional que reciben los proyectos en que han participado. Ramos-Monge y Barrena Martínez (2019) ratifican la importancia de la responsabilidad social universitaria en este tipo de iniciativas como parte del modelo formativo institucional de la universidad actual. Si la institución no comparte esta visión del aprendizaje basado en el civismo y la colaboración social, esta metodología se convierte en una más, dificultando la extensión de los beneficios del proyecto en la comunidad. Los resultados señalan que se trata de una dificultad importante durante la realización de los proyectos, por lo que es necesaria una labor de sensibilización institucional y de planificación de recursos para que los proyectos puedan generar los resultados esperados. 
2) La necesidad de formación didáctica en la metodología en sí es un aspecto resaltado por los participantes, que una vez tienen formación inicial en diferentes áreas de conocimiento, carecen de conocimiento didáctico sobre la metodología para mejor llevarla a cabo como docentes. La formación y actualización pedagógica es un tema relevante en el contexto de la educación superior, puesto que los docentes procedentes de áreas de conocimiento ajenas al campo de la pedagogía necesitan formación didáctica para realizar su trabajo. Así, como ratifican Para y De Ruta 2015, el trabajo con proyectos de ApS debe estar en el proyecto formativo de la universidad y no ser una propuesta individual. Para ello, es importante que la universidad también se implique en analizar y promover capacitaciones docentes si es necesario.

3) Existe una motivación y conciencia sobre los beneficios de aplicar ApS en la educación superior. Los profesores consideran haber adquirido y desarrollado competencias socioemocionales, organizativas y técnicas con el desarrollo de proyectos basados en esta metodología. La consciencia del profesorado sobre la adquisición y/o desarrollo de competencias y la mejora de su práctica a partir de la participación en proyectos de ApS es un estímulo para impulsar proyectos de esta naturaleza.

4) La formación específica para la planificación y gestión de proyectos de ApS es una de las necesidades mencionadas por los participantes. Si la universidad quiere innovar y proporcionar una formación integral, la formación continuada del profesorado es un hito decisivo de gran valor estratégico. En este sentido, el respaldo de la universidad resulta vital, puesto que el docente carece de las herramientas y recursos para afrontar este desafío de forma autónoma. A modo de conclusión, por tanto, los proyectos de ApS han de ser concebidos como una propuesta institucional y como una iniciativa aislada de una asignatura o profesor.

La presente investigación aporta orientaciones en relación con el diseño de acciones formativas para el trabajo con ApS en la Educación Superior tomando como base las competencias que más adquieren, las necesidades formativas y las competencias básicas destacadas por los investigadores sobre el tema.

\section{CONCLUSIONES}

De acuerdo al trabajo presentado, al análisis y discusión, y a los resultados obtenidos, se pueden plantear las siguientes conclusiones principales:

1) El profesorado incide en el deficiente soporte institucional en los proyectos en que han participado.

2) La necesidad de formación didáctica en la metodología en sí es un aspecto resaltado por los participantes

3) Existe una motivación y conciencia sobre los beneficios de aplicar ApS en la educación superior.

4) La formación específica para la planificación y gestión de proyectos de ApS es una de las necesidades mencionadas por los participantes.

\section{REFERENCIAS}

Alvariñas, M., y Fernández-Villarino, M. A., Percepciones de futuros maestros ante nuevos contextos de aprendizaje. Formación universitaria, 4(4), 37-46 (2011).

Arifin, H. M., The Influence of Competence, Motivation, and Organisational Culture to High School Teacher Job Satisfaction and Performance, International Education Studies, 8(1), 38-45 (2015)

Bardin, L., Análisis de contenido, Ediciones Akal, Madrid, España (1991)

Basinger, N., y Bartholomew, K., Service-learning in nonprofit organizations: Motivations, expectations, and outcomes. Michigan Journal of Community Service Learning, 12(2), 15-26 (2006).

Bergsmann, E., Schultes, M. T., y otros tres autores, Evaluation of competence-based teaching in higher education: From theory to practice, Evaluation and program planning, 52, 1-9 (2015).

Briede, J. C., y Mora, M. L., Diseño y co-creación mediante aprendizaje y servicio en contexto vulnerable: análisis de percepción de la experiencia. Formación universitaria, 9(1), 57-70 (2016).

Cabanach, C., Moll, C., y Rodrigo, O. Aprendizaje-servicio: una nueva metodología para formar profesionales del equipo de enfermería. Metas de enfermería, 12(6), 8-12 (2009).

Cano E., Conceptualización y evaluación de competencias docentes, En Cisneros y otros., Evaluación de Competencias Docentes En La Educación Superior, México, Juan Pablos Editor, Distrito Federal, México (2012).

Carbonell, J., y Carrillo, I., Prácticas de cooperación en planes de formación inicial. la educación en valores como vivencia. Aprendizaje servicio y responsabilidad social de las Universidades, 151 (2008).

Cecchi, N. Aprendizaje servicio en Educación Superior. La experiencia latinoamericana. Seminario Internacional Responsabilidad Social Universitaria: Aprendizaje Servicio, 4 (2006). 
Daynes, G., y Longo, N. V., Jane Addams and the origins of service-learning practice in the United States. Michigan Journal of Community Service Learning, 11(1), 5-13 (2004).

De Miguel, M, Cambio de paradigma metodológico en la Educación Superior. Exigencias que conlleva, Cuadernos de Integración Europea, 2, 16-27 (2005).

Dolgon, C., Mitchell, T. D., y Eatman, T. K. (Eds.), The Cambridge Handbook of Service Learning and Community Engagement, Cambridge University Press, Cambridge, Reino Unido (2017).

Folgueiras, P., Luna, E., y Puig, G., Aprendizaje y servicio: estudio del grado de satisfacción de estudiantes universitarios, Revista de Educación, 362, 159-185 (2013).

Furco, A., Is Service-Learning Really Better than Community Service? en Furco, A. y Shelley, H. Billing (Eds), ServiceLearning: The Essence of the Pedagogie, 23-49. Information Age Publishing, Greenwich, Reino Unido (2005).

Gelmon, S. B., Holland, B. A., y Spring, A., Assessing service-learning and civic engagement: Principles and techniques. Stylus Publishing, Herdnon, Estados Unidos (2018).

González-Aldea, P., y Marta-Lazo, C., La metodología del aprendizaje-servicio como herramienta en la formación de los periodistas. Opción, 31(3), 564-581 (2015).

González, J., Wagenaar, R., y Beneitone, P., Tuning-América Latina: un proyecto de las universidades, Revista Iberoamericana de Educación, 35(1), 151-164 (2004).

Goodman, A., Joshi, H., Nasim, B., y Tyler, C., Social and emotional skills in childhood and their long-term effects on adult life. Institute of Education, Londres, Inglaterra (2015).

Hernández, R., Fernández, C., y Baptista, M., Metodología de la investigación, McGraw-Hill, Distrito Federal, México (2010).

Howard, J., Service-learning course design workbook (2001).

Kaye, M. A., The Complete guide to Service Learning. Proven, practical ways to engage students in civic responsibility, academic curriculum \& social action, Free Spirit Publishing Inc, Minneapolis, Estados Unidos (2004).

Kumpulainen, K., y Lankinen, T., Striving for educational equity and excellence: Evaluation and assessment in Finnish basic education, Miracle of education, 69-82 (2016).

Lazo, C. M., y Aldea, P. G., El aprendizaje-servicio, una herramienta para el desarrollo profesional de la responsabilidad social del periodista. Estudios sobre el mensaje periodístico, 18, 577-588 (2012).

Martínez, B., Martínez, I., Alonso, I., y Geruzaga, M., El aprendizaje-servicio, una oportunidad para avanzar en la innovación educativa dentro de la Universidad del País Vasco. (2013).

Martínez, M., Aprendizaje servicio y responsabilidad social de las universidades. Octaedro-Universitat de Barcelona. Institut de Ciències de l'Educació (2008).

Martínez-Usarralde, M. J., Gil-Salom, D., y Macías-Mendoza, D., Revisión sistemática de Responsabilidad Social Universitaria y Aprendizaje Servicio. Análisis para su institucionalización. Revista mexicana de investigación educativa, 24(80), 149-172 (2019).

Navarro, S. B., Zervas, P., Gesa, R. F., y Sampson, D. G., Developing teachers' competences for designing inclusive learning experiences, Journal of Educational Technology \& Society, 19(1), 17-27 (2016).

Perrenoud, P., Diez nuevas competencias para enseñar, Graó, Barcelona, España (2004).

Puig, J. M., Batlle, R., Bosch, C., y Palos, J., Aprendizaje servicio. Educar para la ciudadanía. Octaedro, Barcelona, España (2007).

Ramos-Monge, E., Llinàs, F. J., y Barrena, J., Catalysts of university social responsibility into strategic planning by thematic analysis and deductive coding, International Journal of Entrepreneurship and Innovation Management, 1-28 (2019).

Ramirez, B., y Pizarro, B., Aprendizaje Servicio, Manual para Docentes UC. Dirección General Estudiantil, Vicerrectoría Académica (2005).

Rodrigues, M. W., Isotani, S., y Zárate, L. E., Educational Data Mining: A review of evaluation process in the e-learning, Telematics and Informatics, 35(6), 1701-1717 (2018).

Rodríguez, M.R., El Aprendizaje-Servicio como estrategia metodológica en la Universidad, Revista Complutense de Educación, 25, 95-113 (2014).

Santos, M. Á., Lorenzo, M. M., y Sotelino, A., Sostenibilidad e institucionalización cautelosa del aprendizaje-servicio en la universidad. XXXVII Seminario Interuniversitario de Teoría de la Educación, Universidad de La Laguna, Tenerife, España (2018).

Schuurman, D., De Marez, L., y Ballon, P., The impact of living lab methodology on open innovation contributions and outcomes, Technology Innovation Management Review, 6(1), 7-16 (2016).

Tapia, M. N., Aprendizaje y servicio solidario: en el sistema educativo y las organizaciones juveniles, Ciudad Nueva, Buenos Aires, Argentina (2006). 
Tryon, E., Stoecker, R., Martin, A., Seblonka, K., Hilgendorf, A., y Nellis, M. The Challenge of Short-Term Service-Learning. Michigan Journal of Community Service Learning, 14(2), 16-26 (2008).

Vallaeys, F., La responsabilidad social universitaria: un nuevo modelo universitario contra la mercantilización, Revista Iberoamericana de Educación Superior, 5(12), 105-117 (2014).

Villa, A., y Poblete, M., Aprendizaje basado en competencias, Universidad de Deusto, Bilbao, España (2008).

Wall, T., Giles, D. E., y Stanton, T., Service Learning and Academic Activism: A Review, Prospects and a Time for Revival. In Access to Success and Social Mobility through Higher Education: A Curate's Egg? 163-176. Emerald Publishing Limited, Bingley, Reino Unido (2018).

Zabalza, M., Guía para la planificación didáctica de la docencia universitaria en el marco del EEES (Guía de guías). Documento de trabajo-Universidad de Santiago de Compostela, Santiago de Compostela, España (2004).

Zaitseva, N. A., Larionova, A. y otros cinco autores, Elaboration of the methodology for assessing the development of managerial competences in university students taught with the use of case-technologies, Eurasia Journal of Mathematics, Science and Technology Education, 13(11), 7339-7351 (2017). 